Die Rolle der Investoren für den ökologischen Strukturwandel

\title{
Trau schau wem
}

\section{Kapitalmärkłe können zu einem ökologischen Strukturwandel beitragen, da sie jungen Unternehmen eine Chance geben. Dies zeigt das Beispiel der erneuerba- ren Energien. Allerdings gelingt dies nur, wenn die zunehmend wichtigeren pri- vaten Investoren bei ihrer Geldanlage geeignete ökologische Kriterien berück- sichtigen. Ein Praxisbeispiel zeigt die Herausforderungen, die sich hieraus auch für die Intermediäre ergeben.}

$\mathrm{D}$

Von Andrew Murphy as ethische Investment hat sich in Deutschland zu einem wahrnehmbaren Teilnehmer am Kapitalmarkt entwickelt. Obwohl sich das nach ethischen Kriterien investierte Vermögen in den zurïckliegenden zwei Jahren vervielfacht hat, liegt es erst bei etwa 4 Prozent des gesamten Investitionsvolumens pro Jahr in Deutschland. In anderen Ländern sieht dies anders aus. So wird Schätzungen zufolge in den USA jeder fünfte Dollar nach ethischen Kriterien investiert. In Großbritannien werden bei 8 bis 10 Prozent der Investitionen Umwelt- und Sozial-Kriterien berïcksichtigt.

Das gestiegene Interesse und die zunehmende Nutzung der alternativen Geldanlage werfen die Schwierigkeit einer Definition auf. So existieren die Begriffe nachhaltiges Investment, umwelt- und sozialverträgliche Geldanlage oder ethisches Investment nebeneinander. Was wird jeweils darunter verstanden und wie trägt ihre Umsetzung in der Praxis zum Strukturwandel bei?

Das ethische Investment unterliegt strengen ethischen Kategorien. Dementsprechend werden Investitionen strikt ausgeschlossen, die diese Anlagekategorien verletzen. Die Investitionen unterliegen vor allem Ausschlusskriterien. Oft sind aber auch Positivkriterien vorhanden. Die Ausschlusskriterien werden von einem Anlageausschuss oder der Kapitalanlagegesellschaft festgelegt. Beispielsweise gelten bei den Umwelt-Aktiendepots folgende Negativkriterien als Ausschlusskriterium: Es dürfen keine Investitionen in Unternehmen geleistet werden, die Produktion oder Dienstleistung für die Rüstungs- und Waffenproduktion erstellen. Gesellschaften werden abgelehnt, wenn sie weit reichende Geschäftsbeziehungen in und mit Staaten, in denen Menschenrechtsverletzungen stattfinden, unterhalten. Dabei ist auch das Verhalten des Unternehmens gegen- über seinen Mitarbeitern ein wichtiges Bewertungskriterium. Kommt es zur Diskriminierung von Mitarbeiterinnen und Mitarbeitern aufgrund ihrer politischen, religiösen oder weltanschaulichen Überzeugung, so fällt es durch. Bei den Firmen darf es zu keiner Belastungen der natürlichen Umwelt in vermeidbaren Ausmaßen kommen. Auch Tätigkeiten in oder für die Atomindustrie führen zum Ausschluss.

\section{- Ein exemplarisches Fallbeispiel}

Wie schwierig dies ist und zu welcher unterschiedlichen Beurteilung solch ein Sachverhalt führen kann, zeigt das Beispiel der WEDECO AG aus Düsseldorf. Das Unternehmen ist in der Aufbereitung von Wasser tätig. Mit einer neuen Technik soll Wasser durch UV-Bestrahlung desinfiziert werden. Im bisher gängigen Verfahren wird dafür Chlor verwendet. Das Substitut war dem Chloreinsatz in vielen Punkten überlegen. Es war effizienter und kostengünstiger. Das Produkt ist also ökonomisch und ökologisch eine "Vorzeige"-Investition. Der Aktienkurs hat sich seit Anfang 2000 verfünffacht. Allerdings werden für die Umwelt Aktiendepots bei der Beurteilung des Unternehmens und seines Produktes auch die Einsatzgebiete zur Beurteilung herangezogen. Und da die WEDECO ihre Technik für zwei französische Atomkraftwerke adaptierte und sich an weiteren Ausschreibungen beteiligt, wurde das Investment aus ethisch-ökologischen Gründen abgelehnt. Der Anlageausschuss sah den Einsatz zur Desinfektion des Kühlwassers in Atomkraftwerken als elementaren Bestandteil derselben und somit als Ausschlusskriterium. Der Anlageausschuss des Ökovision Fonds entschied hingegen anders.

Festgelegte Positivkriterien können sein, dass in regenerative Energien und Umwelttechnik investiert wird. Diese werden aber in der Regel sehr weit ausgelegt. Die umwelt- und sozialverträgli- che Geldanlage untersucht die Auswirkungen einer Investition für Umwelt und die Stakeholdergruppen, insbesondere für Arbeitnehmer, Kunden, Lieferanten und die lokale Bevölkerung. Nur wenn eine Investition zu einem Fortschritt für die Umwelt und alle Beteiligten führt, findet eine Anlage statt. Die nachhaltige Anlage trägt dem Grundgedanken Rechnung, den nachfolgenden Generationen die gleichen Entwicklungschancen zu lassen. In der Praxis haben sich SustainabilityAnlagen jedoch als teilweise deckungsgleich mit konventionellen Investitionen erwiesen. So können Investitionen in die Automobilindustrie konform mit den Anlagekriterien sein, sofern das Investitionsziel, wie etwa beim Dow Jones Sustainability Index, als das am nachhaltigsten arbeitende Unternehmen seiner Branche geratet wird. Bei enger ausgelegten Kriterien fallen hingegen Unternehmen aus der Automobilindustrie durch. Über den nachhaltigen Mehrwert einer Investition in Johnson\&Johnson, NOKIA oder BMW kann gestritten werden; eine solche macht wohl nur aus Portfolioausrichtung Sinn.

Ein gutes Beispiel für alle Formen der alternativen Geldanlage sind die regenerativen Energien. Anders als bei der herkömmlichen Energiegewinnung aus Kohle oder Mineralöl tendieren - unter Nachhaltigkeitskriterien ausgedrückt - die "Schulden" gegenüber nachfolgenden Generationen bei den regenerativen Energien gegen Null. Regenerative Energien nutzen die freien Kräfte

\section{Umweltkosten-} management und

Kommunikation - Region Nachbaltigkeit

sind die Themen der Informationsdienste

\section{Ökologisches Wirtschaften $6 / 01$ und $1 / 02$}

Wenn Sie potenzielle Beiträge haben, wenden Sie sich bitte an die Redaktion. 
von Sonne, Wind und Wasser. Und somit entsteht ein Zusatzgewinn, ohne die Belastungen und Gefahren für Umwelt und Menschen.

\section{- Private Rente - neve Marktmacht}

Im Rahmen der Reform der Rentenversicherung in Deutschland wird dem Arbeitnehmer durch die staatliche Bezuschussung der privaten Altersvorsorge in Zukunft eine zunehmende Marktmacht entstehen. Der erwartete Milliardenzufluss in Aktienfonds kann - wenn die Fonds Umwelt- und Sozialkriterien berücksichtigen - eine erhebliche Lenkungsfunktion bedeuten.

Allerdings werden diese Anlageformen wohl hauptsächlich von den etablierten Banken und Kapitalanlagegesellschaften aufgegriffen. Somit jenen, die sich in der Vergangenheit einzig und allein dem Shareholder-Value-Ansatz zur Vermehrung des investierten Geldes verpflichtet gefühlt haben. In $\mathrm{Zu}$ kunft werden es deshalb diese Banken und Kapitalanlagegesellschaften schwer haben, neben ihren konventionellen Aktienfonds auch jene zu managen, die den Belangen der Arbeitnehmer und der Umwelt wahrhaft verpflichtet sind. Wahrscheinlicher ist, dass die auf den Markt kommenden Altersvorsorgefonds in ihrer Anlagephilosophie nur oberflächlich Umwelt- und Sozialkriterien folgen werden. Die vorhandenen Fondsmanagement- und Analysestrukturen bei Banken und Investmentgesellschaften lassen keine andere Möglichkeit zu. Die sich anbietende Alternative für Anleger sind Finanzdienstleister, die ihre Beratung und Angebote auch unter Umwelt- und Sozialkriterien prïfen. Die Symbiose aus einer verantwortungsbewussten Anlagephilosophie und qualifizierter Beratung ermöglichen in Deutschland derzeit nur wenige spezialisierte Finanzdienstleister.

Bereits heute hat sich für die Anleger neben den strukturierten Anlageprodukten mit einer Direktinvestition in Form einer Kommanditbeteiligung eine weitere Anlageform durchgesetzt. Hier investiert der Anleger direkt in ein Projekt, etwa eine Beteiligung an einem Windpark. Dabei leistet der Anleger eine aktiven Beitrag zur weiteren Ökologisierung der Gesellschaft. Interessant wurde diese Form der Investition für Investoren mit einem höheren Einkommen. Sie können bei einer direkten Investition in Windparks ihre Investitionssumme zum Teil steuerlich geltend machen. Allerdings sind Kommanditbeteiligungen erheblich riskanter, was eine genaue Prüfung durch die Anleger erfordert.

\section{Fazit}

Das Beispiel der erneuerbaren Energien hat gezeigt, dass der Kapitalmarkt eine wichtige Rolle für die Weiterentwicklung des ökologischen Strukturwandels besitzt. Er ist in der Lage, Geldströme zu lenken. Junge Unternehmen haben heute die Gelegenheit, sich über den Kapitalmarkt Geld zu besorgen. Dabei werden alte Gesetzmäßigkeiten außer Kraft gesetzt. Entschieden früher die Kreditabteilungen der Banken über das Gelingen oder Misslingen von Unternehmensideen, so erschließt die Börse heute jungen Wachstumsunternehmen eine Investitionsmöglichkeit abseits der Versicherungsund Bankinteressen. Über die Auswahl ihrer privaten Altersvorsorge können zukünftig die Arbeitnehmer selbst darauf Einfluss nehmen, dass ihre Rechte und der ökologische Strukturwandel zunehmend Gestalt annehmen.

Festzuhalten bleibt, dass der ökologische Strukturwandel durch die Unterstiitzung des Kapitalmarktes an Fahrt aufgenommen hat. Die Entwicklung ist noch am Anfang. Eine Unterstiitzung der Politik und der Wissenschaft, sei es direkt oder in Form von Wissenstransfer muss kurz- bis mittelfristig weiter zunehmen. Die Diskrepanz zwischen ethisch/ökologischem gesellschaftlichen Bewusstsein und ethisch/ökologischer Geldanlage geht nirgends so weit auseinander wie in Deutschland. Der regulierende Charakter der Politik muss von der Öffentlichkeit aufgegriffen und weiter umgesetzt werden. Sicherlich haben in der Vergangenheit vor allem Internet-, Medien und Biotechnikunternehmen Nutzen aus der neuen Finanzierungsmöglichkeit etwa durch den Neuen Markt gezogen, aber in deren Schatten haben auch die Umweltunternehmen die Chancen genutzt. Unternehmen wie SolarWorld oder Umweltkontor sind ein Produkt der gewachsenen Risikobereitschaft in Deutschland. Wichtig wird es sein, weitere Unternehmen auf den Markt zu bringen, die den ökologischen Strukturwandel auf breite Schultern stellen. Die Zeit läuft für solche Unternehmen! Bleibt nur zu hoffen, dass die Konkurrenz - die etablierten Großunternehmen - bald aufwachen und es so zu einer gesellschaftlichen Weiterentwicklung kommt.

\section{Der Autor}

Andrew Murphy ist Mitgesellschafter der Researchgesellschaft und Vermögensberatung Murphy\&Spitz Umwelt Consult (www.murphyandspitz.de). Kontakt: Murphy\&Spitz Umwelt Consult, Römerstraße 173, 53117 Bonn. Tel. 0228/ 9676-400, Fax -402, E-mail: andrewmurphy@murphyandspitz.de

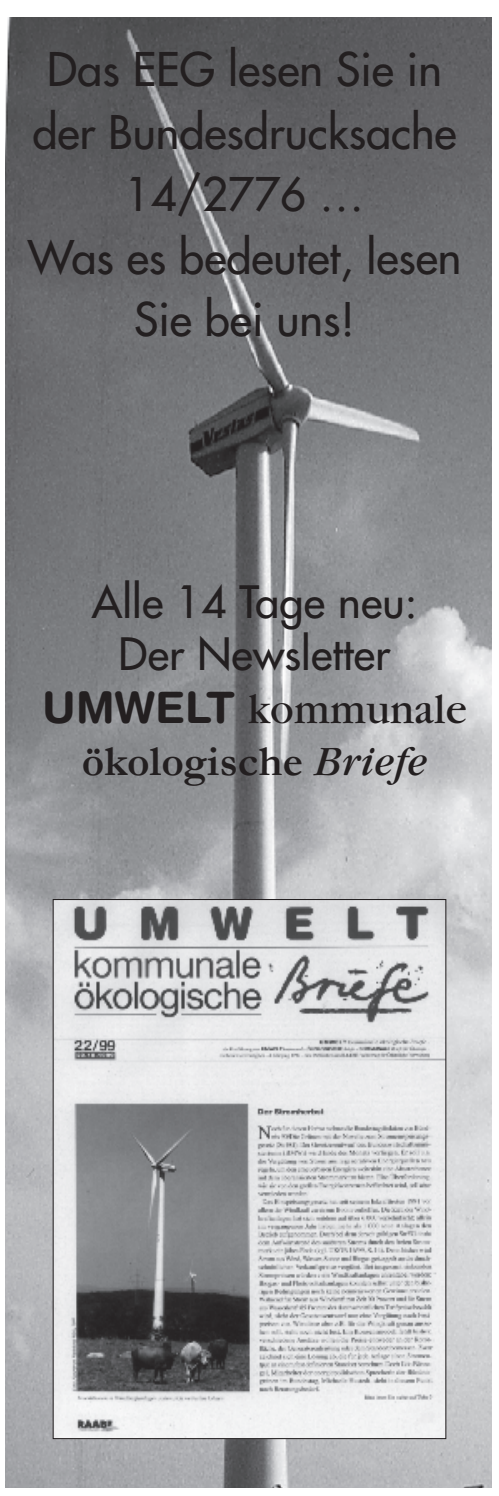

Fordern Sie gleich ein kostenloses Probeabo an!

Schicken Sie den Coupon bitte an:

UmweltBriefe, Raabe Fachverlag Kaiser-Friedrich-Str. 90, D-10585 Berlin Fon 030/212987-23 Fax 030/212987-20

E-mail UMWELTBRIEFE@raabe.de

Internet http://www.umweltbriefe.de

Ja, senden Sie mir die nächsten drei Ausgaben von UMWELT kommunale ökologische Briefe kostenlos zu. Wenn Sie ein Woche nach Erhalt des letzten Freeexemplares nichts von mir Jahr, UMWELT kommunale ökologische Briefe. Der Jahresbezugspreis für ein Abonnement (26 Ausgaben) beträgt $360 \mathrm{DM}$ (unverb. Preisempf.) zzgl. Versandspesen.

Name:

Straße:

PLZ, Ort:

Datum. Unterschrift*):

Ich bin/wir sind berechtigt, die Bestellung des Abonnements ohne Angaben von Gründen innerhalb einer Woche nach Erhalt der drei Freiexemplare in schriftlicher form zu widerrufen. Zur Wahrung der Frist genügt die rechtzeitige Absedung des Widerrufs (Poststempel).

Datum: Unterschrift*):

*) Bitte an beiden Stellen unterschreiben! 
(c) 20I0 Authors; licensee IÖW and oekom verlag. This is an article distributed under the terms of the Creative Commons Attribution Non-Commercial No Derivates License (http://creativecommons.org/licenses/by-nc-nd/3.o/), which permits unrestricted use, distribution, and reproduction in any medium, provided the original work is properly cited. 